\title{
Descripción Anatómica de Cinco Órganos Internos del Delfín Nariz de Botella (Tursiops truncatus), a Través de la Técnica de Plastinación
}

\author{
Anatomy Description of the Five Internal Organs of the Bottlenose \\ Dolphin (Tursiops truncatus) through Plastinated Technique
}

María Dolores Contreras Villanueva*; Roberto Moreno Colín* \& Gabriela Sánchez Fabila*

CONTRERAS, V. M. D.; MORENO, C. R. \& SÁNCHEZ, F. G. Descripción anatómica de cinco organos internos del delfín nariz de botella (Tursiops truncatus), a través de la técnica de plastinación. Int. J. Morphol., 33(2):571-579, 2015.

RESUMEN: Se plastinaron y describieron cinco órganos internos de delfín nariz de botella (Tursiops truncatus) obteniendo modelos didácticos, inodoros, no tóxicos y perdurables que permiten el arreglo de sus componentes en un contexto inalterado; los especímenes resultantes son opacos y firmes pero no irrompibles, con apariencia similar al estado vivo. Las descripciones anatómicas estuvieron en conformidad con los de los autores presentados dentro de los antecedentes de este estudio, afianzando el conocimiento de que los órganos internos de T. truncatus aquí descritos tipifican a los de los mamíferos en aspectos generales ya que se revela gran uniformidad entre sus elementos estructurales. Por otra parte existen pocos trabajos de anatomía no patológica en delfines, y si bien es cierto que se les han realizado varios estudios morfométricos y conductuales, aún puede decirse que muchos rasgos biológicos de los delfines nariz de botella quedan por ser descritos.

PALABRAS CLAVE: Plastinación; Delphinidae; Nariz de Botella; Órganos; Descripción.

\section{INTRODUCCIÓN}

Uno de los avances más relevantes en la preservación de organismos fue realizado mediante el remplazo de los fluidos remanentes en un cuerpo por medio de un polímero. Esta técnica fue ideada en 1977 por el Dr. Gunther von Hagens, que llamó a este proceso "plastinación"; el modelo final obtenido con esta técnica conserva flexibilidad y un color realista sin olor ni toxicidad además de presentar ventajas como que permite disponer y utilizar todo el material de preparados para distintos fines con una mayor durabilidad y limpieza sin tener que descartar piezas que se deterioran con el transcurso de los años cuando son mantenidas con técnicas convencionales.

La disposición de dichos especímenes plastinados ha asistido ampliamente en la comprensión de la anatomía de los organismos ya que estas muestras naturales son muy valiosas para la comprensión cabal de las complejas estructuras tridimensionales en sus respectivas posiciones e interrelaciones (Ali \& Al-Thnaian, 2007), tarea ardua con el uso exclusivo de libros o fotografías por buena que sea su calidad (Reyes Aguilar, 2007); mientras que dichas muestras plastinadas permiten el arreglo de todos los componentes del tejido para ser estudiados en un contexto inalterado (von Hagens et al., 1987).

Para los biólogos es muy importante contar con nuevos conocimientos de diferentes grupos de animales como los mamíferos los cuales deben su espectacular triunfo biológico a muchas características adaptativas (Norris, 1977). Por ejemplo el orden de los Cetáceos está formado por un conjunto de mamíferos adaptados a la vida marina, gracias a la morfología, anatomía y fisiología tan específicas adquiridas a lo largo del camino evolutivo (Castelló-Orvay, 1976).

Todos son por completo acuáticos; la caja torácica alberga y protege a los órganos internos. Tienen la capacidad de alternar periodos de eupnea y apnea; la capacidad de los cetáceos para seguir activos durante largos periodos de apnea depende quizá de varias adaptaciones en las vías respiratorias (Vaughan, 1988). 
Los Odontocetos constituyen el suborden de mayor importancia entre los Cetáceos en términos de abundancia, diversidad de especies y distribución (Vaughan), siendo la familia Delphinidae el grupo más abundante y diverso.

Tursiops truncatus es una especie cosmopolita distribuida en todos los océanos templados y tropicales del mundo siendo la especie de mamífero marino costal más común en el Golfo de México (Waring et al., 2000).

El objetivo del trabajo fue de describir anatómicamente cinco órganos de delfín nariz de botella Tursiops truncatus y procesarlos a través de la técnica de plastinación con la finalidad de que se cuente con material didáctico y se puedan seguir estudiando y observando sin que se deteriore con el tiempo.

\section{MATERIAL Y MÉTODO}

Se trabajó con 5 órganos internos del delfín $T$. truncatus (1 pulmón, 1 tracto reproductor (femenino), 1 lengua y 2 corazones) colectados y etiquetados por el Dr. Alberto Delgado Estrella y el Biol. Arturo Romero Tenorio en Cancún, Quintana Roo, gracias a un convenio de colaboración entre la Facultad de Estudios Superiores Iztacala U.N.A.M y el Instituto Vía Delphi para la Investigación de los Mamíferos Acuáticos A.C. (del parque ecológico Xcaret).

Dichas muestras se recibieron directamente en el Laboratorio de Anatomía Animal Comparada (FES-I) por medio de paquetería en contenedores de plástico envueltos e impregnados con formol. Los órganos se lavaron en agua corriente dentro de cubetas para en seguida tomar las medidas morfométricas de cada uno; posteriormente fueron sumergidos en formaldehído al 4\% durante 7 días en recipientes de plástico a temperatura ambiente con el fin de fijarlos y evitar su descomposición.

Deshidratación. Transcurrido este tiempo nuevamente se sumergieron en agua corriente durante 2 días para seguidamente comenzar con el periodo de deshidratación de un mes en acetona al $100 \%$ a $-30{ }^{\circ} \mathrm{C}$; durante este tiempo se realizaron varios cambios de acetona con el fin de eliminar residuos de grasa y agua del órgano, siendo reemplazados por acetona.

Impregnación. Posteriormente inició la impregnación pasiva, realizada gradualmente con los órganos saturados en acetona llevándolos a resina poliéster mc-40 en dos etapas que duraron de uno a tres meses cada una a temperatura ambiente:
- Inmersión en una solución 1:1 de resina y acetona (en cubetas de plástico con tapa).

- Impregnación en resina 100\% (en frascos de vidrio).

Secado. Cumplido este lapso se removió el exceso de resina lavando los órganos en acetona pura, una vez secos se rociaron con esmalte acrílico de secado rápido dándoles de 2 a 3 aplicaciones con un periodo de secado de 3 a 15 días. Finalmente plastinados los distintos órganos se tomaron nuevamente sus medidas para iniciar las descripciones anatómicas.

\section{RESULTADOS}

Fueron obtenidas las medidas morfométricas de los órganos internos del delfín nariz de botella (T. truncatus) antes y después del proceso y con ellas se calculó el porcentaje de deformación de cada órgano (Tabla I) por medio de la siguiente fórmula:

$\%$ de deformación $=(\mathrm{y} 0-\mathrm{yf} / \mathrm{y} 0)$ x 100; En donde: $\mathrm{y} 0=$ medida inicial, $\mathrm{yf}=$ medida final

Se observó la retención de varias características posteriores al proceso, como: color, rigidez, estructura anatómica, tamaño, flexibilidad y detalle de superficie (Tablas I y II).

El detalle de superficie se refiere a la calidad del aspecto externo del órgano una vez plastinado mientras que la estructura anatómica se relaciona con la conservación de la morfología de los especímenes después de la plastinación (Mayoral Robles, 2010).

En todos los casos se observó un cambio de tonalidad de rosado/rojizo a café obscuro que ocurrió durante el proceso de impregnación pasiva (Fig. 1A y 1B); así mismo, se advierte la reducción del tamaño original de los órganos de $4 \%$ hasta $11 \%$ de largo y de $6 \%$ hasta $16 \%$ de ancho (Tabla I). En cuanto a la forma cabe mencionar que todos los órganos conservaron su aspecto original (Fig. 1), y pese a que adquirieron notable rigidez mantuvieron una textura cercana a la del órgano en fresco.

Tabla I. Cálculo del porcentaje de deformación de los órganos.

\begin{tabular}{lcc}
\hline Órgano & Largo & Ancho \\
\hline Lengua & $4 \%$ & $16 \%$ \\
Corazón 1 & $11 \%$ & $6 \%$ \\
Corazón 2 & $8 \%$ & $6 \%$ \\
Tracto Reproductor $(-)$ & $7 \%$ & $13 \%$ \\
\hline
\end{tabular}


Tabla II. Características de los órganos internos de T. truncatus plastinados.

\begin{tabular}{lcccc}
\hline Órgano & Color & Rigidez & $\begin{array}{c}\text { Detalle de } \\
\text { Superficie }\end{array}$ & $\begin{array}{c}\text { Est ructura } \\
\text { Anatómica }\end{array}$ \\
\hline Lengua & Negruzco & Sí & Bueno & Nomal \\
Corazón 1 & Negruzco & Sí & Bueno & Normal \\
Corazón 2 & Negruzco & Sí & Bueno & Normal \\
Pulmón & Negruzco & Sí & Bueno & Normal \\
Tracto Reproductor & Negruzco & Sí & Bueno & Normal \\
\hline
\end{tabular}
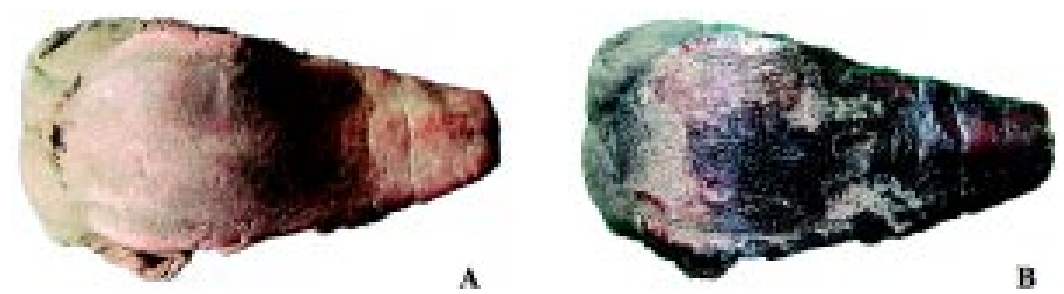

Fig. 1. Lengua de T. truncatus fijada en formol 4\% (A) y plastinada (B).
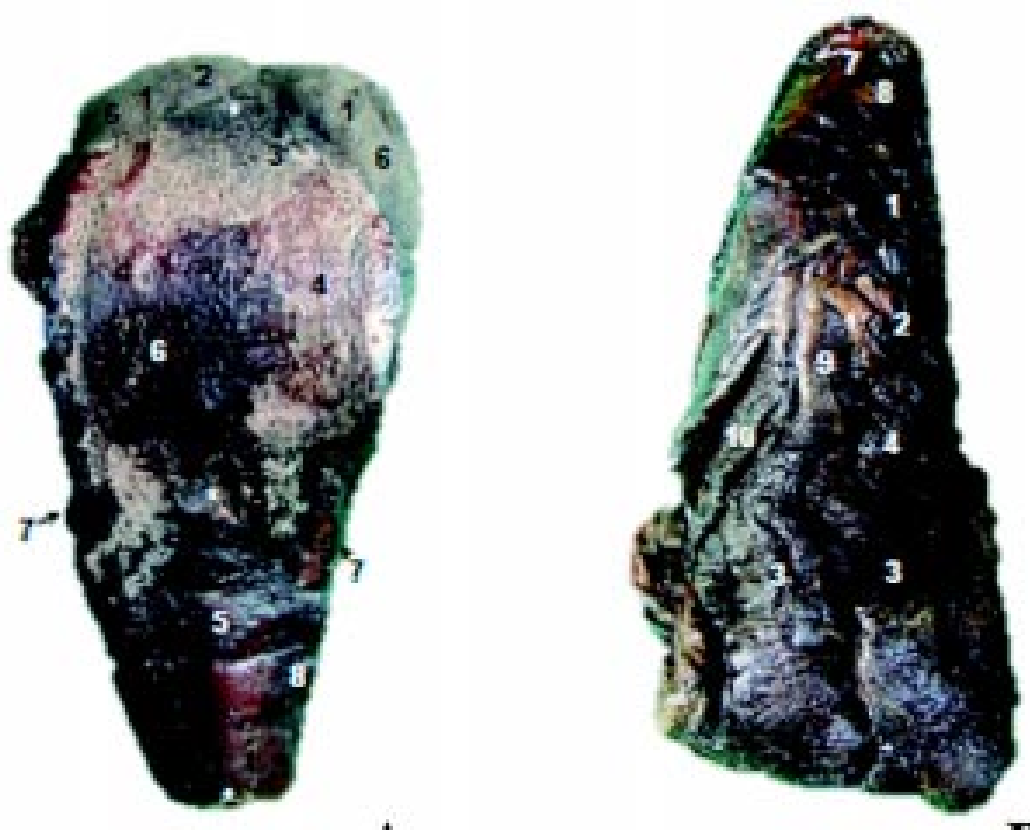

A

B

Fig. 2. A. Vista superior del dorso y la raíz de la lengua. 1. Folículos linguales; 2. Dorso de la lengua, porción postsulcal (post.); 3. Surco terminal; 4. Dorso de la lengua, porción presulcal (ant.); 5. Surco medio; 6. Poros gustativos; 7. Remanentes de papilas marginales; 8 Margen de la lengua. B. Vista inferior de los músculos linguales. 9. Músculo palatogloso; 10. Músculo estilogloso; 11. Músculo geniogloso; 12. Músculo hiogloso; 13. Frenillo lingual; 14. Pliegue fimbriado; 15. Tabique lingual; 16. Músculo longitudinal inferior.

\section{Descripción anatómica de los órganos} trabajados.

Lengua. Es de forma cónica, tiene un tamaño de $13,7 \mathrm{~cm}$ de largo por $4,3 \mathrm{~cm}$ de ancho y un peso de $92,7 \mathrm{~g}$. Presenta remanentes de papilas gustativas marginales (Figs. 1 y 2) (Kastelein \& Dubbeldam, 1990).

La superficie de la lengua (Fig. 2A) es lisa, sin embargo se encuentra jaspeada por multitud de pequeños poros gustativos (Fig. 2B) exceptuando en el margen (Sokolov \& Volkova, 1973). En la raíz de la lengua (Fig. 2A) se encuentran los surcos terminales, que son dos surcos linguofaríngeos angulados de manera que forman una amplia $\mathrm{V}$ con el ápice dirigido hacia la faringe. No se observa un foramen ciego (foramen caecum) [9].

Se observan entre otros, a los músculos hiogloso y estilogloso (Fig. 2B) encargados de la retracción de la lengua, así como el músculo longitudinal inferior responsable del acortamiento y ensanchamiento lingual (Donaldson, 1977).

Corazón. El corazón número 1 (Fig. 3) es ancho, corto y romo; con $8,7 \mathrm{~cm}$ de largo por $10,8 \mathrm{~cm}$ de ancho y un peso de 122,1 g. El corazón número 2 difiere en cuanto a forma y dimensiones, ya que tiene un tamaño de $14,8 \mathrm{~cm}$ de largo por $11,2 \mathrm{~cm}$ de ancho y un peso de $273,1 \mathrm{~g}$. Se les describen dos caras, dos márgenes y un ápice.

El corazón está comprimido dorsoventralmente, su cara ventral es convexa y su cara dorsal plana (Fig. 2B). Dorsalmente y a la derecha llegan las venas cavas al atrio derecho y tres venas pulmonares unidas en un tronco común al atrio izquierdo (Fig. 3C). La cara ventral del corazón se halla dividida por el surco interventricular paraconal (Fig. 3D) que surge del surco coronario hacia la izquierda y que divide completamente a la parte ventricular de esta cara en los dos ventrículos, hacia la derecha el ventrículo derecho y hacia la izquierda el ventrículo izquierdo (Pérez \& Lima, 2006). 


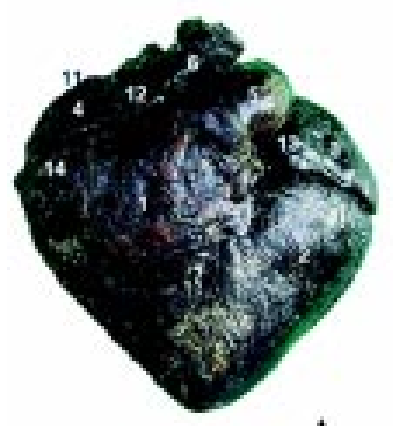

$\mathbf{A}$

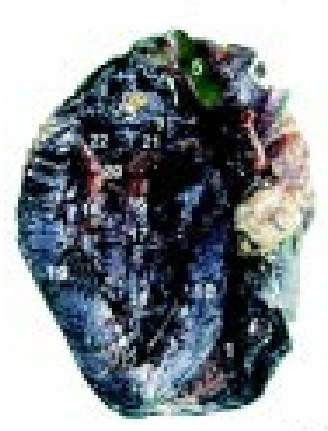

C

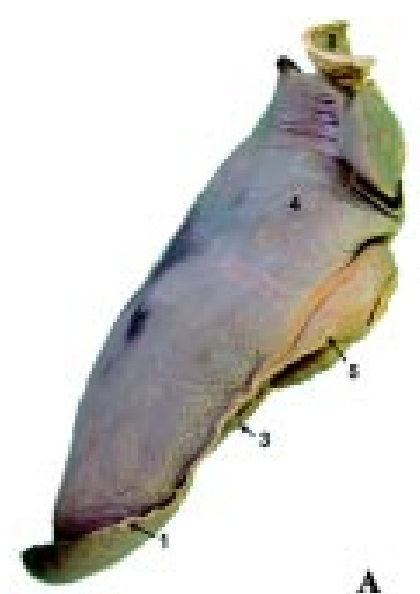

$\mathbf{A}$

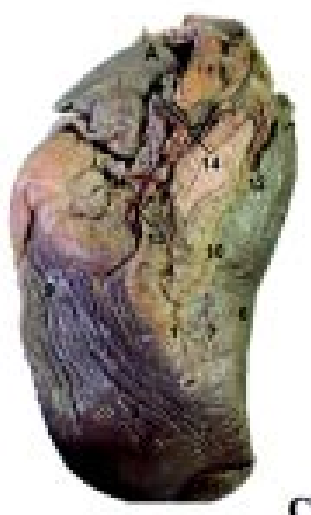

C

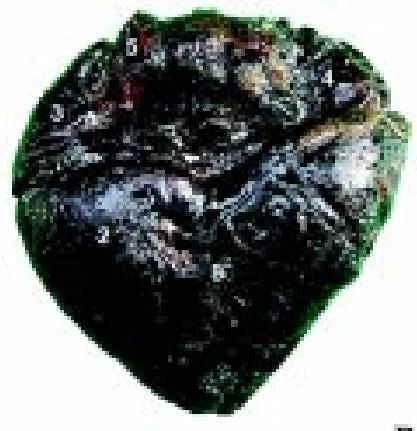

B

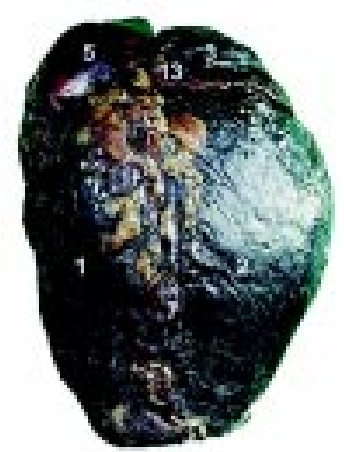

D

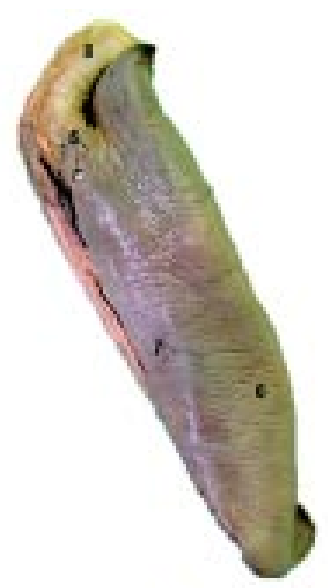

B

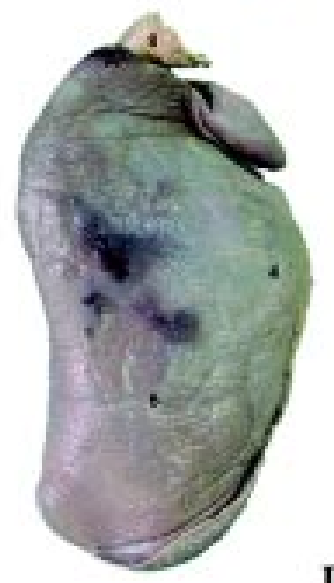

D
Fig. 3. Corazón 1: (A) vista anterior y (B) posterior. Corazón 2: (C) configuración interna y (D) vista anterior. 1. Ventrículo derecho; 2. Ventrículo izquierdo; 3 . Atrio izquierdo; 4. Atrio derecho; 5. Tronco pulmonar; 6 Parte ascendente de la aorta; 7 Surco y rama interventricular paraconal; 8. Surco y rama interventricular subsinual; 9. Ostio atrioventricular izquierdo; 10. Septo interventricular; 11. Surco terminal; 12. Aurícula derecha; 13. Aurícula izquierda; 14 . Venas cardiacas anteriores; 15. Músculo papilar anterior; 16. Músculo papilar posterior; 17. Trabécula septomarginal; 18. Fibras de Purkinje; 19. Pared del ventrículo izquierdo; 20 Cuerdas tendinosas de la valva atrioventricular izquierda; 21 . Cúspide anterior de la valva atrioventricular izquierda; 22. Cúspide posterior de la valva atrioventricular izquierda.

El surco coronario se encuentra interrumpido en el nivel de emergencia del tronco pulmonar (Fig. 3A). En la cara atrial este surco divide completamente atrios de ventrículos. El surco interventricular subsinusal (Fig. 3C) divide completamente la parte ventricular de la cara atrial en los dos ventrículos situados hacia los costados respectivos del animal (Pérez \& Lima). Ambos surcos interventriculares se continúan entre sí en el ápex del corazón. Los márgenes ventriculares derecho e izquierdo se sitúan, respectivamente, a la derecha y a la izquierda del animal. Ambos constituyen los márgenes derecho e izquierdo del contorno cardiaco y son ligeramente oblicuos de lateral a medial llegando al ápice. La longitud promedio de estos márgenes es de $6,0 \mathrm{~cm}$, desde el surco coronario hasta el ápice, que corresponde a ambos ventrículos (Pérez \& Lima).

Pulmón. Los pulmones son estructuras elásticas, esponjosas, que se encuentran fijas al mediastino por el hilio pulmonar, a través del cual entran y salen los vasos arteriales y venosos y los grandes bronquios.

Fig. 4. Pulmón Derecho: (A) vista ventral, (B) vista dorsal, (C) cara interna y (D) cara lateral. A. Ápex; 1. Margen basal; 2. Margen ventral; 3. Superficie diafragmática; 4, Cara lateral de la superficie costal; 5. Bronquio traqueal; 6. Cara horizontal de la superficie costal; 7. Impresión esofágica; 8. Tráquea; 9. Impresión cardiaca; 10. Margen dorsal; 11. Margen caudal; 12. Impresión vascular; 13. Procesos papilares relacionados con los bronquios; 14. Procesos papilares relacionados con los bronquios traqueales. 
Se describe un pulmón con peso de $2,13 \mathrm{~kg}$, longitud de $31,3 \mathrm{~cm}$ y ancho de $23,5 \mathrm{~cm}$. Muestra una forma elíptica, con un ápice agudo craneal (Fig. 4A) y un extremo caudal en punta (Fig. 4B). Se reseñan tres superficies: costal, medial y diafragmática.

La superficie costal (Fig. 4B) exhibe una cara lisa y sin fisuras interlobulares; esta superficie presenta un margen longitudinal obtuso cráneo-caudal (Fig. 4C) que separa una cara dorsal horizontal plana de una cara lateral convexa. Mientras que la superficie medial (Fig. 4D) presenta las piezas mediales y mediastinales; en ella se observa una impresión cardiaca (4C) pero no así la muesca cardiaca. En tanto que la superficie diafragmática (Fig. 4C) es oblicua (Concha, 2011).

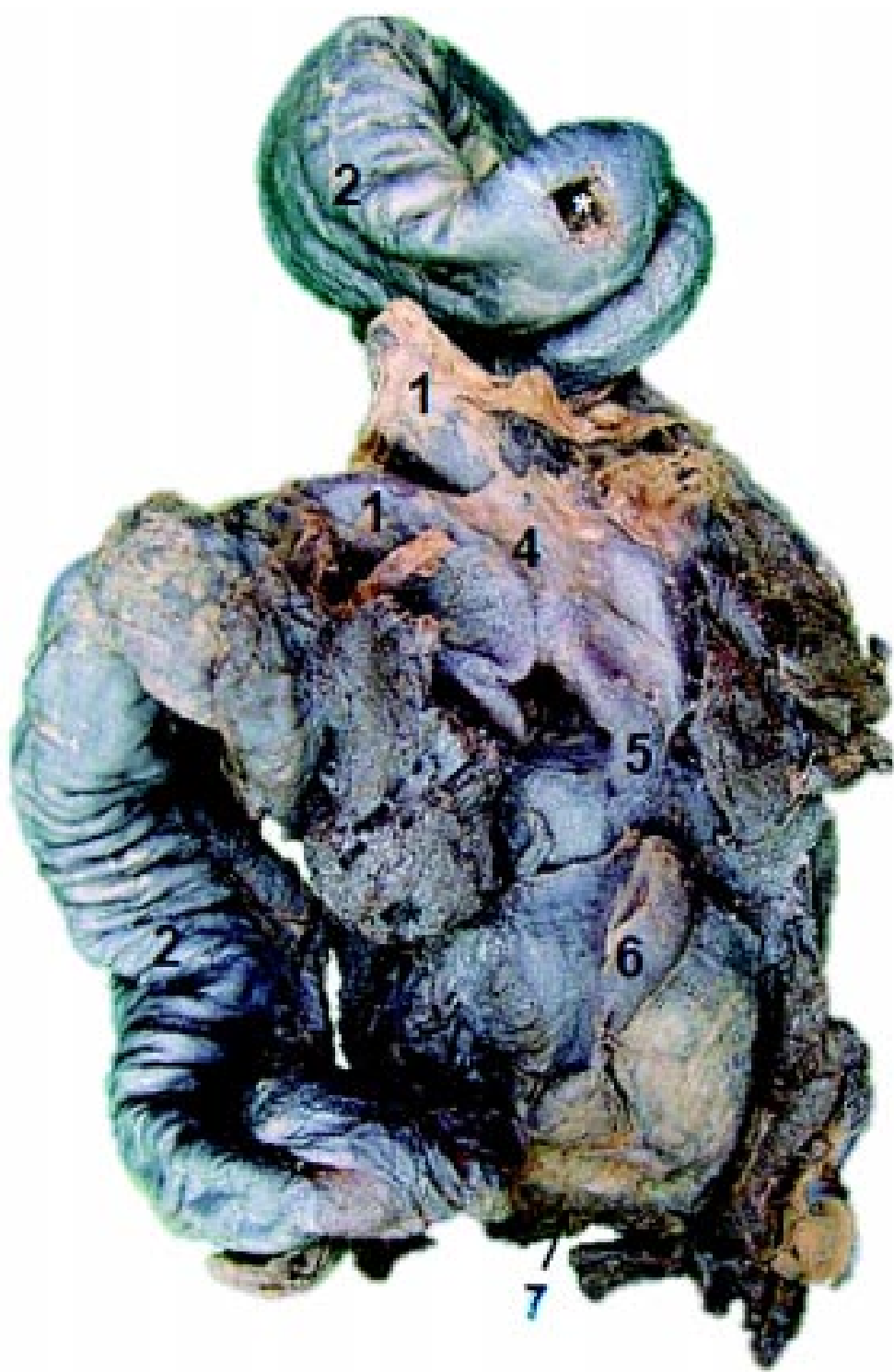

Fig. 5. Cara anterior del útero. 1. Cuernos uterinos; 2. Tubas uterinas; 3. Ovario; 4. Cuerpo uterino; 5. Cérvix; 5. Vagina; 7. Apertura vaginal.
En el pulmón derecho, se visualiza un surco para el esófago y para las venas cava caudal y craneal así como cuatro procesos papilares dos de ellos relacionados con el hilio craneal (traqueal) (Fig. 4C) y los dos restantes con el hilio principal (Fig. 4D) (Concha).

Tracto reproductor de la hembra. Tiene un peso de $452 \mathrm{~g}$; como en el caso de todos los cetáceos, se compone de un útero bicorne con un cuerpo uterino comparativamente más pequeño y dos cuernos (Fig. 5A) (Norris) que se unen posteriormente formando un útero corto (Donaldson). Desde el cuerpo uterino estos cuernos se dirigen primero en dirección craneal y después en dirección lateral. Ambos cuernos y cuerpo uterino están provistos de numerosos pliegues longitudinales; los conductos que constituyen los cuernos y tubas uterinas se encuentran más bien retorcidos (Norris). La tuba uterina derecha tiene una longitud aproximada de $24,6 \mathrm{~cm}$ mientras que la izquierda es de $27,2 \mathrm{~cm}$.

Los ovarios (Fig. 5), ovoides y ligeramente suaves (Berta et al., 2006), están situados en la cara dorsal proximal de la tuba uterina, que termina en un útero que desciende sin fusión para abrir justo sobre la os uterina en una pequeña parte de la matriz común a ambos tubos. En la parte inferior de la vagina se observa una pequeña apertura entre esta y la vulva (Fig. 5); el esfínter muscular vaginal está bien desarrollado envolviendo el tubo en esta región, y en condiciones usuales prácticamente cierra el pasaje (Meek, 1918).

\section{DISCUSIÓN}

Fue aplicada la técnica de plastinación modificada en cinco órganos internos de delfín nariz de botella utilizando resina poliéster mc40 y como anotaba Von Hagens los especímenes resultantes con este tipo de resinas son opacos y firmes pero no irrompibles, técnica adecuada para elaborar piezas de demostración como las del presente estudio.

Las piezas biológicas obtenidas ofrecen las ventajas de ser duraderas, almacenables y manipulables en seco sin medida preventiva, con 
forma y volumen natural, textura aproximada a la real, inodoros y no desprenden vapores tóxicos. Dichos especímenes no precisan de tratamientos especiales para su mantenimiento y se pueden mantener limpios por métodos tradicionales.

Los órganos se describieron destacando su estructura específica, permitiendo el estudio de la función anatómica y textura que suelen perderse con la técnica de preservación típica en formol. Pese a que los órganos plastinados mantuvieron sus formas y estructuras en la posición anatómica esperada, las desventajas recaen en que sus dimensiones se redujeron de manera poco significativa, perdieron flexibilidad y color original del mismo modo que se reporta en los trabajos de Silva Pérez (2007) y Ali \& Al Thnaian.

Así mismo, los órganos plastinados probablemente perdieron su color original durante el proceso, debido a que durante la acción del polímero y la acetona, los pigmentos de los cromatóforos fueron expuestos y por consiguiente, diluidos como señalara Silva Pérez. Del mismo modo al explicar este ennegrecimiento deben ser consideradas tanto la oxidación de la hemoglobina como la posibilidad de que sea resultado del índice de refracción de la resina que Von Hagens \& Whalley (2009), destacaron acerca de sus primeros ensayos sobre plastinación. Lo anterior tomando también en consideración que la fijación es un paso fundamental que no se llevó a cabo de primera instancia en el Laboratorio de Anatomía Animal Comparada, debido a que los órganos fueron enviados envueltos en gasas impregnadas en formol. Por otra parte, Miklosová \& Miklos (2004), mencionaron que los órganos que no alcanzan a impregnarse en su totalidad tienden a encogerse adquiriendo un color obscuro. Todo esto ligado a la omisión del proceso de curado (Peroxido de metil etil cetona y acetona), durante el cual la emulsificación atenúa el color del especimen.

La forma de los órganos se mantuvo ya que los especímenes no mostraron diferencias perceptibles en la calidad del detalle de superficie, aunque se produjo una reducción dimensional acaso atribuible al efecto de fijación y deshidratación y quizá a un rápido permeado del especimen en un solo baño de resina/acetona antes de entrar a resina pura.

La pérdida de flexibilidad de los tejidos se imputa al uso de resina poliéster mc-40 en lugar de alguno de los silicones BIODUR ${ }^{\circledR}$ típicamente utilizados para la técnica estándar de plastinación, esto debido a que como mencionan Daviau et al. (1997), es necesario contar con un permiso para realizar la técnica con los materiales originalmente dispuestos para este fin. Ya Von Hagens señalaba que los especímenes plastinados con copolímeros epoxi- silicona resultan lo suficientemente rígidos como para ser pulidos pero no son irrompibles; aunque plastinar con este tipo de resinas ofrece la ventaja práctica de poder llevar a cabo la impregnación forzada a temperatura ambiente. Todo esto considerando del mismo modo lo anotado por Ripani et al. (1994), acerca de que el tiempo de deshidratación debe ser el menor posible, para prevenir la fragilidad y pérdida de flexibilidad de los especímenes.

Las estructuras de cada órgano se mantuvieron tal como en los esquemas consultados para su descripción, no obstante, al no haber obtenido los órganos del delfín dentro del LAAC, hay estructuras que no se presentan en la descripción. La única dificultad durante la descripción anatómica inherente al proceso de plastinación se debió al cambio de coloración del órgano que en ocasiones impedía distinguir claramente las estructuras. Otro obstáculo que se presentó fue que algunos órganos tenían algunas estructuras plegadas incluso antes de comenzar con la técnica (y por tanto ajeno al proceso), ocultando así otras secciones del órgano.

Se presentaron incidentes durante el plastinado de los corazones y el estómago, donde la resina se polimerizó antes del término del proceso. Lo anterior sucedió estando los órganos en solución resina/acetona (1:1) y en resina pura; y esto pudiera explicarse tomando en cuenta que la impregnación a temperatura ambiente limita el tiempo de vida de la resina, debido a que la viscosidad del polímero se incrementa después de algunas semanas. Por otra parte, se reduce el tiempo de impregnación como apuntan Ripani et $a l$. Este problema se pudiera resolver con la sugerencia de Kularbkaewi et al. (1996), mediante la mezcla del polímero con acetona antes de rehusarlo en un nuevo baño de especímenes.

Debido a estos percances los órganos se retiraron de las soluciones en las que se encontraban y se llevaron a un baño de acetona ( 4 días en el caso de los corazones y 6 en el del estómago), durante el cual se fueron eliminando manualmente los trozos de resina sobrante, cuando fue necesario esto se hizo con ayuda de una fresadora (Dremel) para el corazón número 2 , así como lijas bastardas y aguja de disección en el estómago, hasta limpiar casi en su totalidad los órganos antes mencionados.

Como mencionan Donaldson y Sokolov \& Volkova, en relación con lo referente a la lengua de $T$. truncatus, una de sus características más notables es la ausencia de papilas filiformes, fungiformes, foliadas o circunvaladas presentes en la mayoría de los mamíferos, por lo que podría ser que el gusto sea un sentido quimiorreceptor en Odontocetos. De la misma forma que los autores anteriormente señalados, tam- 
bién en el presente trabajo se hallaron surcos terminales que anteriormente no se describían en el T. truncatus. En estudios anteriores se advierte la existencia de algunos quimiorreceptores que pudieran funcionar como receptores gustativos, siendo la raíz de la lengua el lugar más probable para encontrarlos.

La presencia de remanentes de papilas marginales sugiere lo señalado por Kastelein \& Dubbeldam referente a que las papilas tienen la función de impedir la filtración de leche hacia la cavidad oral durante el periodo de lactancia, así como evitar que el agua expulsada al ingerir el alimento vuelva a ingresar a la cavidad oral cuando el delfín comienza a alimentarse de peces. Dichas papilas reducen su tamaño con el paso del tiempo, por tanto no se observan tan claramente en el especimen plastinado. Otros mecanismos para limitar el paso del agua hacia el interior como lo señala Donalson en la acción conjunta de los músculos estilogloso, hiogloso y longitudinal inferior que parecen permitirle a la lengua ayudar a sellar la orofarínge; así como en la observación de que los delfines pueden expulsar enérgicamente chorros de agua desde la cavidad oral probablemente presionando firmemente la lengua contra la mandíbula superior. Indudablemente la musculatura de la lengua juega un rol fundamental en la alimentación del delfín, ya que como destaca dicho autor empuja la comida hacia la orofarínge, orientando el pez para ser tragado siempre comenzando por la cabeza (probablemente para que las aletas y cola no se atoren). Finalmente el hecho de que los labios del delfín no sean móviles aunado a la presencia de papilas marginales en delfines jóvenes hace factible que la lengua sea vital en el proceso de succión.

La anatómica cardiaca macroscópica del corazón de T. truncatus coincide con la descripción hecha por Pérez \& Lima en el corazón del delfín del Plata (Pontoporia blainvillei) así como con la de los atlas de anatomía humana consultados. Sin embargo, destaca el caso del corazón número 2 en el que de la misma manera reseñada por Powell et al. (2009) se observa una transposición de las arterias pulmonar y aorta, hipertrofia del ventrículo izquierdo, así como una pequeña conexión entre ambos atrios en forma de orificio (defecto ventricularseptal) no perceptible en las fotografías exhibidas dentro de los resultados. Como se menciona en dicho estudio existen pocas condiciones descritas en humanos y animales domésticos que expliquen anomalías cardiacas y vasculares múltiples, siendo una de estas condiciones la llamada Tetralogía de Fallot caracterizada por una aorta predominante, obstrucción del flujo del ventrículo derecho, defecto ventricular septal e hipertrofia del ventrículo derecho. Colectivamente estos defectos causan una reducción del flujo sanguíneo hacia los pulmones permitiendo a la vez la mezcla de sangre proveniente de ambos flancos del corazón, dando lugar a la afluencia de sangre pobre en oxígeno desde el corazón hacia el resto del cuerpo.

Alusivo al pulmón como señalaron Berta et al. existen diferencias importantes entre los pulmones de mamíferos terrestres y marinos. La anatomía externa del pulmón de T. truncatus se equipara a la que Concha realizó sobre los pulmones de Phocoena spinipinnis, y del mismo modo que dicho autor indica para esta marsopa, el pulmón de T. truncatus tampoco presenta fisuras interlobulares en su superficie costal, dato que ya indicaba Berta et al. en algunos Pinípedos, sugiriendo una tendencia a reducir la lobulación. Lógicamente esta característica impidió identificar y señalar lóbulos en la descripción del presente estudio. Por otra parte, hay que estimar que aparentemente el gran tamaño de los pulmones de delfínidos y focoenidos es una condición derivada que parece permitirles funcionar como un almacén de oxígeno para el intercambio gaseoso respiratorio de estos activos buceadores de aguas relativamente someras.

Lo reseñado acerca de la anatomía hepática de $T$. truncatus es similar a lo expuesto en los atlas de anatomía humana consultados.

La estructura anatómica del bazo de $T$. truncatus concuerda con la que Berta et al. realizó en Pinípedos y Cetáceos; así como con la reportada en los atlas de anatomía humana consultados. Respecto a lo reportado por Colangelo et al. (2006), relativo a la función del bazo en Cetáceos, estos autores señalaron como controvertida ya que el pequeño tamaño de este órgano, junto con su típica forma globular y superficie lisa, pueden argumentar en contra de su función de almacenamiento de glóbulos rojos oxigenados.

Los estómagos de los Cetáceos mantienen un sistema uniforme de morfología estomacal con las excepciones señaladas por Mead (1993) de las familias Ziphiidae (zifios) y Platanistidae (delfines de río). El estómago del $T$. truncatus, difiere únicamente en tamaño respecto al de una ballena azul (Balaenoptera musculus).En el caso del delfín nariz de botella, como en todos los Cetáceos, se habla de un estómago plurilocular que consiste en: ante-estómago, estómago principal, cámaras de conexión y dos compartimentos pilóricos.

El delfín nariz de botella, como todos los mamíferos marinos, posee un riñón reniculado como señalan Lauria de Cidre \& Klein (1994) y Berta et al., lo que según estos últimos autores permite la producción de grandes volúmenes de orina concentrada. Para explicar esta característica 
se debe considerar lo indicado por Lauria de Cidre \& Klein al aseverar que los mamíferos marinos desarrollaron esta reniculación como un recurso evolutivo presionados por el medio en cuanto a la economía del agua, permitiéndoles albergar un mayor número de nefronas en un volumen renal menor, además de facilitar el funcionamiento independiente de cada renículo.

La anatomía externa del órgano reproductor de la hembra de T. truncatus es similar a lo reportado por Meek en la marsopa(Phocoena communis), por Berta et al. sobre los Pinípedos, así como con las descripciones generales sobre el útero no preñado en Cetáceos realizadas por Norris y Plön \& Bernard (2007). Se observa que los órganos genitales son simples y típicos siguiendo el patrón general del útero bicorne en mamíferos. Sin embargo, es de interés lo señalado por Meek acerca de la angosta entrada a la vagina que puede ser una adaptación para ocluir la entrada de agua hacia la cavidad de la vaginal.
De esta manera, los resultados anatómicos estuvieron en concordancia con los autores consultados en este estudio, lo cual afianza el conocimiento de que los órganos internos de T. truncatus aquí descritos tipifican a los de los mamíferos en aspectos generales.

Cabe destacar lo mencionado por Daviau et al., que el proceso de plastinación está actualmente en uso para la creación de modelos para escuelas médicas y veterinarias, aplicándose en una amplia variedad de especies, con la única limitación del tamaño de la cámara de impregnación; aunque la mayor desventaja radica en la necesidad de obtener licencia de Von Hagens para utilizar la técnica patentada y obtener los materiales, motivo por el cual son necesarias más investigaciones adaptando la técnica original para crear muestras permanentes, de buena calidad, y de alto carácter ilustrativo con un costo implícito menor, como este trabajo que tuvo como objetivo ayudar a la instrucción de la anatomía general de algunos órganos de T. truncatus.

CONTRERAS, V. M. D.; MORENO, C. R. \& SÁNCHEZ, F. G. Anatomy description of the five internal organs of the bottlenose dolphin (Tursiops truncatus) through plastinated technique. Int. J. Morphol., 33(2):571-579, 2015.

SUMMARY: Five internal organs of bottlenose dolphin (Tursiops truncatus) were plastinated and described, obtaining odorless, nontoxic and perdurable didactic models, which let their components to be modified in an unaltered context; the resulting specimens are opaque and firm, but not unbreakable, with an appearance similar to that of the living state. The anatomic descriptions were made in concordance with those of the authors presented within the antecedents of this study; reinforcing the knowledge that the internal organs of T. truncatus described here typify those of mammals in general aspects, since the great uniformity between their structural elements is revealed. Moreover, there are very few non pathological anatomic studies about dolphins; even though various mophometric and behavioral studies have been carried out, it still can be considered that several biological aspects of bottlenose dolphins remain to be described.

KEY WORDS: Plastination; Delphinidae; Bottle nose; Organs; Description.

\section{REFERENCIAS BIBLIOGRÁFICAS}

Ali, A. M. \& Al-Thnaian, T. A. Preservation of ruminant and equine anatomical specimens by silicone plastination. Sci. J. King Faisal Univ., 8(1):111-9, 2007.

Berta, A.; Sumich, J. L. \& Kovacs, K. M. Marine Mammals: Evolutionary Biology. 2a ed. San Diego, Academic Press, 2006. p.547.

Waring, G. T.; Quintal, J. M. \& Swartz, S. L. (Eds.). US Atlantic and Gulf of Mexico marine mammal stock assessments -- 2000. Massachusetts, US Dep Commer, NOAA Tech Memo NMFS NE 162, 2000.

Castelló-Orvay, F. Morfología, anatomía y biología de los cetáceos. Inmers. Cienc., (10-11):133-43, 1976.
Colangelo, P.; Marruchella, G.; Cozzi, B.; Mazzariol, S.; García Hartmann, M.; Stanzani, G.; Fichtel, L.; Olivieri, V.; Albanese, V. \& Di Guardo, G. Accesory spleens in cetaceans: A morphopathological study in three bottlenose dolphins (Tursiops truncatus) and one Risso's dolphin (Grampus griseus). Poster en Congreso. Cattolica, Conferencia Internacional de Cetáceos, Tortugas Marinas y Tiburones del Mar Adriático, 2006.

Concha, I. Anatomical description of the lungs of Burmeister's porpoise (Phocoena spinipinnis). Santiago de Chile, Anato.cl, 2011. Disponible en: http://www.anato.cl/0007proyectos/ porpoise/IConcha\%20AAVA\%20UST2011-porpoise.pdf

Daviau, J. S.; Parker, J. C.; Parmelee, R. H.; Jahn, S. E. \& Frank, D. A. The use of plastinated specimens as teaching aids of orolaryngeal anatomy in selected laboratory animals. Contemp. Top Lab. Anim. Sci., 36(6):50-2, 1997. 
Donaldson, B. J. The tongue of the bottlenosed dolphin (Tursiops truncatus). In: Harrison, R. J. (Ed.). Functional Anatomy of Marine Mammals. Vol. 3. New York, Academic Press, 1977. p.428.

Kastelein, R. A. \& Dubbeldam, J. L. Marginal papillae on the tongue of the Harbour porpoise (Phocoena phocoena), Bottlenose dolphin (Tursiops truncatus) and Commerson's dolphin (Cephalorhynchus commersonii). Aquat. Mamm., 15(4):158-70, 1990.

Kularbkaewi, C.; Cook, P.; Yutanawiboonchai, W.; Von Hagens, G. Plastinated Pathology Specimens at Room Temperature in Thailand. J. Int. Soc. Plastination; 11(1):17-20, 1996.

Lauria de Cidre, L. \& Klein, M. Observaciones morfofuncionales en el riñón del lobo marino de un pelo Otaria flavescens. Misc. Zool., 17:213-23, 1993-1994.

Mayoral Robles, S. Comparación entre la impregnación forzada a$25{ }^{\circ} \mathrm{C}$ y a temperatura ambiente en el proceso de plastinación de órganos de animales. Tesis de Licenciatura (Medicina Veterinaria y Zootecnia). México D. F., Facultad de Medicina Veterinaria y Zootecnia, UNAM, 2010. p.39.

Mead, J. G. The systematic importance of stomach anatomy in beaked whales. Int. Mar. Biol. Res Ins.: IBI Rep., 4:75-86, 1993.

Meek, A. The Reproductive Organs of Cetacea. J. Anat., 52(Pt. 2):186-210, 1918.

Miklosová, M. \& Miklos, V. Plastination with silicone method S 10-monitoring and analysis causes of failure. Biomed. Pap. Med. Fac. Univ. Palacky Olomouc Czech Repub., 148(2):237-8, 2004.

Norris, K. S. Whales, dolphins and porpoises. Berkeley, University of California Press, U.S.A., 755-89, 1977.

Pérez, W. \& Lima, M. Cardiac Anatomy of the Pontoporia blainville. Int. J. Morphol., 24(3):351-6, 2006.

Plön, S. \& Bernard, R. T. F. Anatomy with Particular Reference to the Female. In: Miller, D. L. (Ed.). Reproductive Biology and Phylogeny of the Cetacea: Whales, Dolphins and Porpoises. Enfield, Science Publishers, 2007. p.428.

Powell, J. W.; Archibald, R. T.; Cross, C. A.; Rotstein, D. S.; Soop, V. M. \& McFee, W. E. Multiple congenital cardiac abnormalities in an Atlantic bottlenose dolphin (Tursiops truncatus). J. Wildl. Dis., 45(3):839-42, 2009.

Reyes Aguilar, M. E. Anatomía humana y plastinación. Bol. Mex. His. Fil. Med., 10(1):34-9, 2007.

Ripani, M.; Bassi, A.; Perracchio, L.; Panebianco, V.; Perez, M.; Boccia, M. L.; Marinozzi G. Monitoring and enhancement of fixation, dehydration, forced impregnation and cure in the standard S-10 technique. J. Int. Soc. Plastination, 8:3-5, 1994.
Silva Pérez, E. Análisis de la Técnica de Plastinación Aplicada a Corazones de Cerdo. Tesis de Licenciatura (Biología). México D. F., Facultad de Estudios Superiores Iztacala, Universidad Nacional Autónoma de México, 2007. pp.42.

Sokolov, V. E. \& Volkova, O. V. Structure of the dolphin's tongue. In: Chapskii, K. K. \& Sokolov, V. E. (Eds.). Morphology and Ecology of Marine Mammals: Seals, Dolphins, Porpoises. New York, Wiley, 1973. p.321.

Vaughan, T. A. Mamíferos. $3^{\mathrm{a}}$ ed. México D. F., Editorial Interamericana, 1988. p.587.

von Hagens, G.; Tiedemann, K. \& Kriz, W. The current potential of plastination. Anat. Embryol. (Berl.), 175(4):411-21, 1987.

von Hagens, G. \& Whalley, A. Body Worlds - The Original Exhibition of Real Human Bodies. Catálogo de la Exhibición. 14a Reimpresión. Heidelberg, Verlagsgesellschaft mbH, 2009. p.281.

Dirección para Correspondencia:

Mtra. Gabriela Sánchez Fabila

FES Iztacala en el laboratorio de Anatomía de vertebrados y Educación Científica LAVEC

Universidad Nacional Autónoma de México

Avenida de los Barrios Número 1

Colonia Los Reyes Iztacala

Tlalnepantla, Estado de México, C.P. 54090

MÉXICO

Email: gsfabila@gmail.com

Recibido: 24-11-2014

Aceptado: 14-04-2015 